\title{
Correction: Nilsson, J. et al. Maximal Q Factor for an On-Chip, Fuse-Based Trimmable Capacitor. Electronics 2019, 8, 62
}

\author{
Joakim Nilsson $^{1, * \mathbb{D}}$, Johan Borg ${ }^{2} \mathbb{D}$ and Jonny Johansson ${ }^{1} \mathbb{D}$ \\ 1 Department of Computer Science, Electrical and Space Engineering, Luleå University of Technology, Luleå \\ 971 87, Sweden; jonny@ltu.se \\ 2 Department of Physics, Imperial College, London SW7 2AZ, UK; j.borg@imperial.ac.uk \\ * Correspondence: joanil@ltu.se
}

Received: 4 June 2019; Accepted: 12 June 2019; Published: 18 June 2019

check for updates

The authors wish to make the following correction to our published paper [1]. In the last expression in Equation (7), as well as the expressions on both sides of the equality sign in Equation (9), the denominators and the numerators have been accidentally swapped. Furthermore, Equation (9) contains an additional typo, where two occurrences of $R_{2}$ have been accidentally substituted by $X_{2}$. In summary, on page 4, Equation (7) should be changed from:

$$
Q_{\mathrm{eq}}=-\frac{\Im\left\{Z_{\mathrm{eq}}\right\}}{\Re\left\{Z_{\mathrm{eq}}\right\}}=-\frac{X_{1}\left(R_{2}^{2}+X_{2}^{2}\right)+X_{2}\left(R_{1}^{2}+X_{1}^{2}\right)}{R_{1}\left(R_{2}^{2}+X_{2}^{2}\right)+R_{2}\left(R_{1}^{2}+X_{1}^{2}\right)}
$$

to the following correct version:

$$
Q_{\mathrm{eq}}=-\frac{\Im\left\{Z_{\mathrm{eq}}\right\}}{\Re\left\{Z_{\mathrm{eq}}\right\}}=-\frac{R_{1}\left(R_{2}^{2}+X_{2}^{2}\right)+R_{2}\left(R_{1}^{2}+X_{1}^{2}\right)}{X_{1}\left(R_{2}^{2}+X_{2}^{2}\right)+X_{2}\left(R_{1}^{2}+X_{1}^{2}\right)} .
$$

Equation (9) should be changed from:

$$
-\frac{X_{1}\left(R_{2}^{2}+X_{2}^{2}\right)+X_{2}\left(r_{\mathrm{f}, \text { on }}^{2}+X_{1}^{2}\right)}{r_{\mathrm{f}, \text { on }}\left(R_{2}^{2}+X_{2}^{2}\right)+X_{2}\left(r_{\mathrm{f}, \text { on }}^{2}+X_{1}^{2}\right)}=-\frac{X_{1}\left(R_{2}^{2}+X_{2}^{2}\right)+X_{2}\left(k_{\mathrm{f}}^{2} r_{\mathrm{f}, \text { on }}^{2}+X_{1}^{2}\right)}{k_{\mathrm{f}} r_{\mathrm{f}, \text { on }}\left(R_{2}^{2}+X_{2}^{2}\right)+X_{2}\left(k_{\mathrm{f}}^{2} r_{\mathrm{f}, \text { on }}^{2}+X_{1}^{2}\right)}
$$

to the following correct version:

$$
-\frac{r_{\mathrm{f}, \text { on }}\left(R_{2}^{2}+X_{2}^{2}\right)+R_{2}\left(r_{\mathrm{f}, \text { on }}^{2}+X_{1}^{2}\right)}{X_{1}\left(R_{2}^{2}+X_{2}^{2}\right)+X_{2}\left(r_{\mathrm{f}, \text { on }}^{2}+X_{1}^{2}\right)}=-\frac{k_{\mathrm{f}} r_{\mathrm{f}, \text { on }}\left(R_{2}^{2}+X_{2}^{2}\right)+R_{2}\left(k_{\mathrm{f}}^{2} r_{\mathrm{f}, \text { on }}^{2}+X_{1}^{2}\right)}{X_{1}\left(R_{2}^{2}+X_{2}^{2}\right)+X_{2}\left(k_{\mathrm{f}}^{2} r_{\mathrm{f}, \text { on }}^{2}+X_{1}^{2}\right)}
$$

The authors would like to apologise for any inconvenience caused to the readers by these changes. The change does not affect the scientific results. The manuscript will be updated and the original will remain online on the article webpage, with reference to this correction.

\section{Reference}

1. Nilsson, J.; Borg, J.; Johansson, J. Maximal Q Factor for an On-Chip, Fuse-Based Trimmable Capacitor. Electronics 2019, 8, 62. [CrossRef]

(C) 2019 by the authors. Licensee MDPI, Basel, Switzerland. This article is an open access article distributed under the terms and conditions of the Creative Commons Attribution (CC BY) license (http:/ / creativecommons.org/licenses/by/4.0/). 